Network Working Group

Request for Comments: 3372

BCP : 63

Category: Best Current Practice
A. Vemuri

Qwest Communications

J. Peterson

Neustar

September 2002

\title{
Session Initiation Protocol for Telephones (SIP-T): Context and Architectures
}

\section{Status of this Memo}

This document specifies an Internet Best Current Practices for the Internet Community, and requests discussion and suggestions for improvements. Distribution of this memo is unlimited.

Copyright Notice

Copyright (C) The Internet Society (2002). All Rights Reserved.

Abstract

The popularity of gateways that interwork between the PSTN (Public Switched Telephone Network) and SIP networks has motivated the publication of a set of common practices that can assure consistent behavior across implementations. This document taxonomizes the uses of PSTN-SIP gateways, provides uses cases, and identifies mechanisms necessary for interworking. The mechanisms detail how SIP provides for both 'encapsulation' (bridging the PSTN signaling across a SIP network) and 'translation' (gatewaying).

Table of Contents

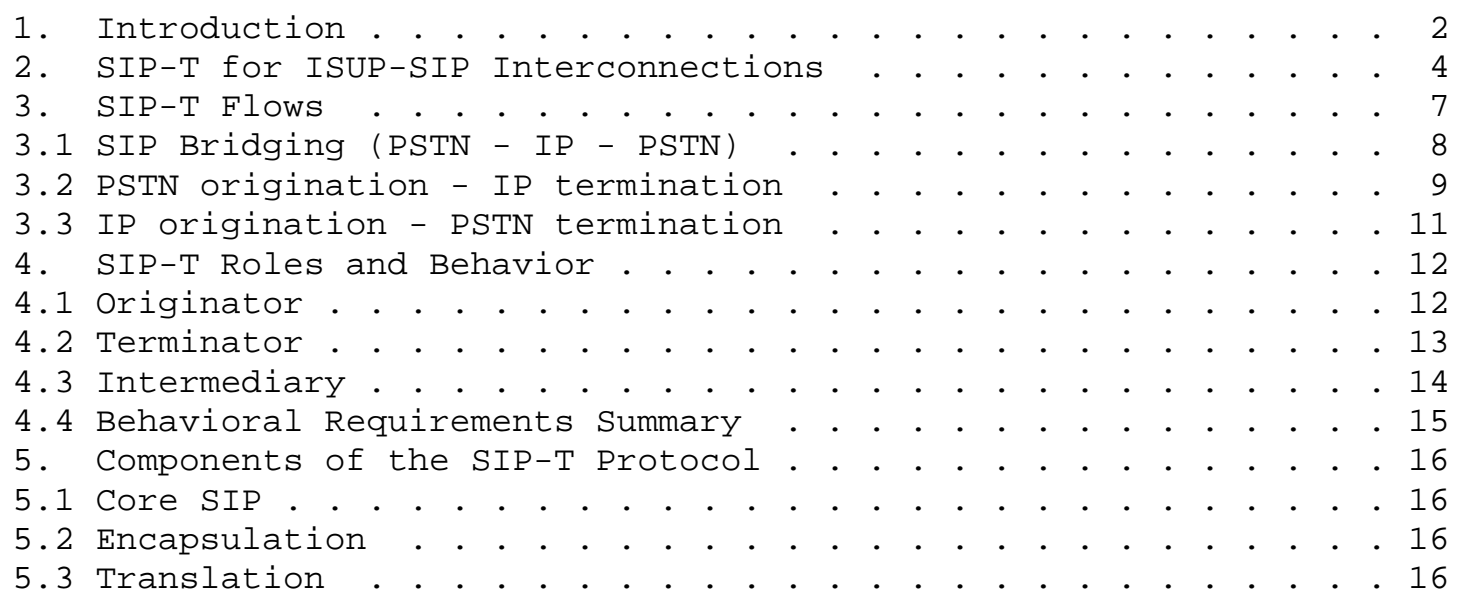


5.4 Support for mid-call signaling . . . . . . . . . . . . . . . . 17

6. SIP Content Negotiation . . . . . . . . . . . . . . . 17

7. Security Considerations . . . . . . . . . . . . . . 19

8. IANA Considerations . . . . . . . . . . . . . . . . 20

9. References . . . . . . . . . . . . . . . . . . . . 20

10 References . . . . . . . . . . . . . . . . . . 20

A. Notes . . . . . . . . . . . . . . . . . . . . 21

B. Acknowledgments . . . . . . . . . . . . . . . . . . 21

Authors' Addresses . . . . . . . . . . . . . . . . . . . . 22

Full Copyright Statement . . . . . . . . . . . . . . . 23

1. Introduction

The Session Initiation Protocol (SIP [1]) is an application-layer control protocol that can establish, modify and terminate multimedia sessions or calls. These multimedia sessions include multimedia conferences, Internet telephony and similar applications. SIP is one of the key protocols used to implement Voice over IP (VoIP).

Although performing telephony call signaling and transporting the associated audio media over IP yields significant advantages over traditional telephony, a VoIP network cannot exist in isolation from traditional telephone networks. It is vital for a SIP telephony network to interwork with the PSTN.

The popularity of gateways that interwork between the PSTN and SIP networks has motivated the publication of a set of common practices that can assure consistent behavior across implementations. The scarcity of SIP expertise outside the IETF suggests that the IETF is the best place to stage this work, especially since SIP is in a relative state of flux compared to the core protocols of the PSTN. Moreover, the IETF working groups that focus on SIP (SIP and SIPPING) are best positioned to ascertain whether or not any new extensions to SIP are justified for PSTN interworking. This framework addresses the overall context in which PSTN-SIP interworking gateways might be deployed, provides use cases and identifies the mechanisms necessary for interworking.

An important characteristic of any SIP telephony network is feature transparency with respect to the PSTN. Traditional telecom services such as call waiting, freephone numbers, etc., implemented in PSTN protocols such as Signaling System No. 7 (SS7 [6]) should be offered by a SIP network in a manner that precludes any debilitating difference in user experience while not limiting the flexibility of SIP. On the one hand, it is necessary that SIP support the primitives for the delivery of such services where the terminating point is a regular SIP phone (see definition in section 2 below) rather than a device that is fluent in SS7. However, it is also essential that SS7 information be available at gateways, the points 
of SS7-SIP interconnection, to ensure transparency of features not otherwise supported in SIP. If possible, SS7 information should be available in its entirety and without any loss to trusted parties in the SIP network across the PSTN-IP interface; one compelling need to do so also arises from the fact that certain networks utilize proprietary SS7 parameters to transmit certain information through their networks.

Another important characteristic of a SIP telephony network is routability of SIP requests - a SIP request that sets up a telephone call should contain sufficient information in its headers to enable it to be appropriately routed to its destination by proxy servers in the SIP network. Most commonly this entails that parameters of a call like the dialed number should be carried over from SS7 signaling to SIP requests. Routing in a SIP network may in turn be influenced by mechanisms such as TRIP [8] or ENUM [7].

The SIP-T (SIP for Telephones) effort provides a framework for the integration of legacy telephony signaling into SIP messages. SIP-T provides the above two characteristics through techniques known as 'encapsulation' and 'translation' respectively. At a SIP-ISUP gateway, SS7 ISUP messages are encapsulated within SIP in order that information necessary for services is not discarded in the SIP request. However, intermediaries like proxy servers that make routing decisions for SIP requests cannot be expected to understand ISUP, so simultaneously, some critical information is translated from an ISUP message into the corresponding SIP headers in order to determine how the SIP request will be routed.

While pure SIP has all the requisite instruments for the establishment and termination of calls, it does not have any baseline mechanism to carry any mid-call information (such as the ISUP INF/INR query) along the SIP signaling path during the session. This midcall information does not result in any change in the state of SIP calls or the parameters of the sessions that SIP initiates. A provision to transmit such optional application-layer information is also needed. 
Problem definition: To provide ISUP transparency across SS7-SIP interworking

SS7-SIP Interworking Requirements

$=================$
Transparency of ISUP

Signaling

Routability of SIP messages with dependencies on ISUP

Transfer of mid-call ISUP signaling messages
SIP-T Functions

Encapsulation of ISUP in the SIP body

Translation of ISUP information into the SIP header

Use of the INFO Method for midcall signaling

Table 1: SIP-T features that fulfill PSTN-IP inter-connection Requirements

While this document specifies the requirements above, it provide mechanisms to satisfy them - however, this document does serve as an framework for the documents that do provide these mechanisms, all of which are referenced in section 5.

Note that many modes of signaling are used in telephony (SS7 ISUP, BTNUP, Q.931, MF etc.). This document focuses on SS7 ISUP and aims to specify the behavior across ISUP-SIP interfaces only. The scope of the SIP-T enterprise may, over time, come to encompass other signaling systems as well.

2. SIP-T for ISUP-SIP Interconnections

SIP-T is not a new protocol - it is a set of mechanisms for interfacing traditional telephone signaling with SIP. The purpose of SIP-T is to provide protocol translation and feature transparency across points of PSTN-SIP interconnection. It intended for use where a VoIP network (a SIP network, for the purposes of this document) interfaces with the PSTN.

Using SIP-T, there are three basic models for how calls interact with gateways. Calls that originate in the PSTN can traverse a gateway to terminate at a SIP endpoint, such as an IP phone. Conversely, an IP phone can make a call that traverses a gateway to terminate in the PSTN. Finally, an IP network using SIP may serve as a transit network between gateways - a call may originate and terminate in the PSTN, but cross a SIP-based network somewhere in the middle. 
The SS7 interfaces of a particular gateway determine the ISUP variants that that gateway supports. Whether or nor a gateway supports a particular version of ISUP determines whether it can provide feature transparency while terminating a call.

The following are the primary agents in a SIP-T-enabled network.

- PSTN (Public Switched Telephone Network): This refers to the entire interconnected collection of local, long-distance and international phone companies. In the examples below, the term Local Exchange Carrier ( $L E C$ ) is used to denote a portion (usually, a regional division) of the PSTN.

- IP endpoints: Any SIP user agent that can act as an originator or recipient of calls. Thus, the following devices are classified as IP endpoints:

* Gateways: A telephony gateway provides a point of conversion between signaling protocols (such as ISUP and SIP) as well as circuit-switch and packet-switched audio media. The term Media Gateway Controller (MGC) is also used in the examples and diagrams in this document to denote large-scale clusters of decomposed gateways and control logic that are frequently deployed today. So for example, a SIP-ISUP gateway speaks ISUP to the PSTN and SIP to the Internet and is responsible for converting between the types of signaling, as well as interchanging any associated bearer audio media.

* SIP phones: The term used to represent all end-user devices that originate or terminate SIP VoIP calls.

* Interface points between networks where administrative policies are enforced (potentially middleboxes, proxy servers, or gateways) .

- Proxy Servers: A proxy server is a SIP intermediary that routes SIP requests to their destinations. For example, a proxy server might direct a SIP request to another proxy, a gateway or a SIP phone. 


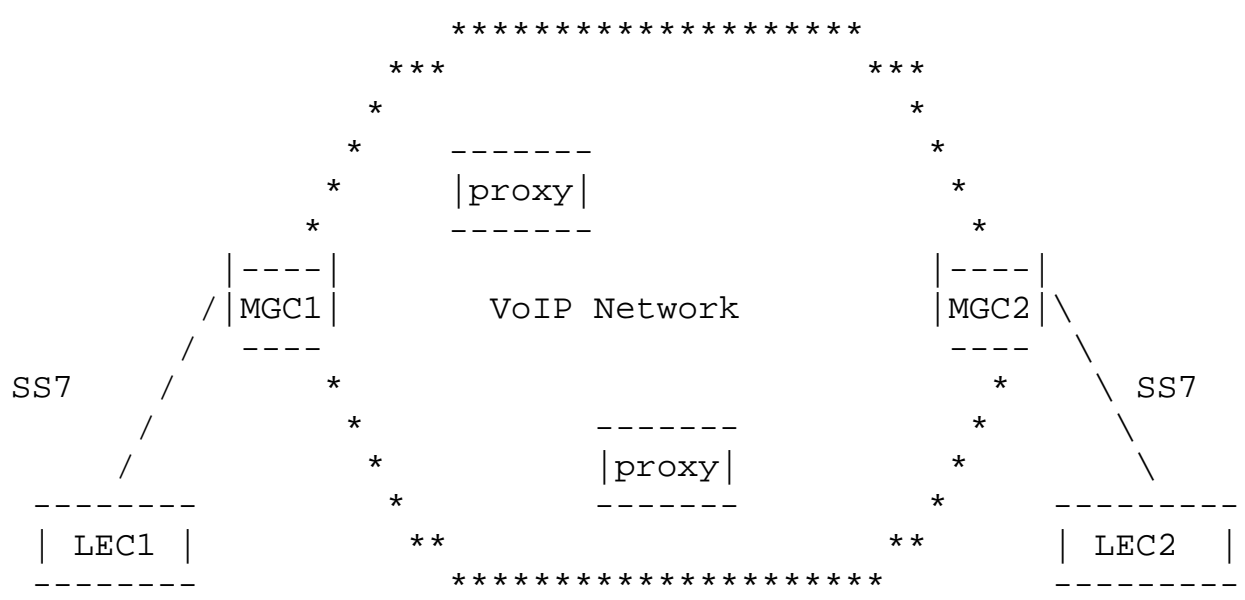

Figure 1: Motivation for SIP-T in ISUP-SIP interconnection

In Figure 2 a VoIP cloud serves as a transit network for telephone calls originating in a pair of LECs, where SIP is employed as the VoIP protocol used to set up and tear down these VoIP calls. At the edge of the depicted network, an MGC converts the ISUP signals to SIP requests, and sends them to a proxy server which in turn routes calls on other MGCs. Although this figure depicts only two MGCs, VoIP deployments would commonly have many such points of interconnection with the PSTN (usually to diversify among PSTN rate centers). For a call originating from LEC1 and be terminating in LEC2, the originator in SIP-T is the gateway that generates the SIP request for a VoIP call, and the terminator is the gateway that is the consumer of the SIP request; MGC1 would thus be the originator and MGC2, the terminator. Note that one or more proxies may be used to route the call from the originator to the terminator.

In this flow, in order to seamlessly integrate the IP network with the PSTN, it is important to preserve the received SS7 information within SIP requests at the originating gateway and reuse this SS7 information when signaling to the PSTN at the terminating gateway. By encapsulating ISUP information in the SIP signaling, a SIP network can ensure that no SS7 information that is critical to the instantiation of features is lost when SIP bridges calls between two segments of the PSTN.

That much said, if only the exchange of ISUP between gateways were relevant here, any protocol for the transport of signaling information may be used to achieve this, obviating the need for SIP and consequently that of SIP-T. SIP-T is employed in order to leverage the intrinsic benefits of utilizing SIP: request routing and call control leveraging proxy servers (including the use of forking), 
ease of SIP service creation, SIP's capability negotiation systems, and so on. Translation of information from the received ISUP message parameters to SIP header fields enables SIP intermediaries to consider this information as they handle requests. SIP-T thus facilitates call establishment and the enabling of new telephony services over the IP network while simultaneously providing a method of feature-rich interconnection with the PSTN.

Finally, the scenario in Figure 2 is just one of several flows in which SIP-T can be used - voice calls do not always both originate and terminate in the PSTN (via gateways); SIP phones can also be endpoints in a SIP-T session. In subsequent sections, the following possible flows will be further detailed:

1. PSTN origination - PSTN termination: The originating gateway receives ISUP from the PSTN and it preserves this information (via encapsulation and translation) in the SIP messages that it transmits towards the terminating gateway. The terminator extracts the ISUP content from the SIP message that it receives and it reuses this information in signaling sent to the PSTN.

2. PSTN origination - IP termination: The originating gateway receives ISUP from the PSTN and it preserves this ISUP information in the SIP messages (via encapsulation and translation) that it directs towards the terminating SIP user agent. The terminator has no use for the encapsulated ISUP and ignores it.

3. IP origination - PSTN termination: A SIP phone originates a VoIP call that is routed by one or more proxy servers to the appropriate terminating gateway. The terminating gateway converts to ISUP signaling and directs the call to an appropriate PSTN interface, based on information that is present in the received SIP header.

4. IP origination - IP termination: This is a case for pure SIP. SIP-T (either encapsulation or translation of ISUP) does not come into play as there is no PSTN interworking.

3. SIP-T Flows

The follow sections explore the essential SIP-T flows in detail. Note that because proxy servers are usually responsible for routing SIP requests (based on the Request-URI) the eventual endpoints at which a SIP request will terminate is generally not known to the originator. So the originator does not select from the flows 
described in this section, as a matter of static configuration or on a per-call basis - rather, each call is routed by the SIP network independently, and it may instantiate any of the flows below as the routing logic of the network dictates.

3.1 SIP Bridging (PSTN - IP - PSTN)

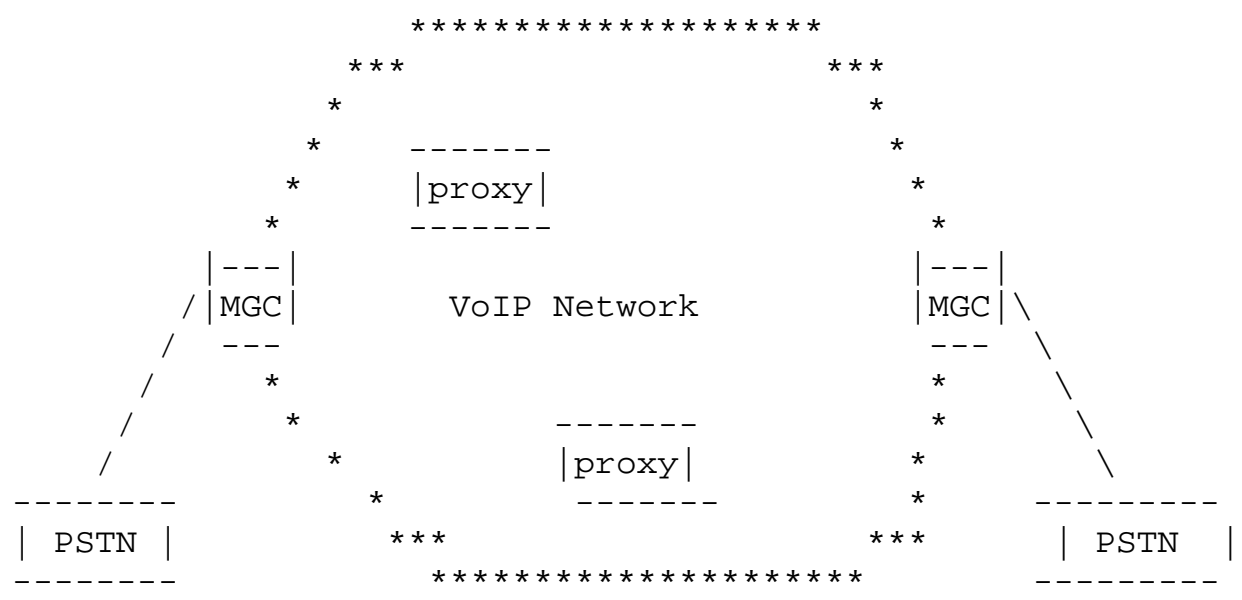

Figure 2: PSTN origination - PSTN termination (SIP Bridging)

A scenario in which a SIP network connects two segments of the PSTN is referred to as 'SIP bridging'. When a call destined for the SIP network originates in the PSTN, an SS7 ISUP message will eventually be received by the gateway that is the point of interconnection with the PSTN network. This gateway is from the perspective of the SIP protocol the user agent client for this call setup request. Traditional SIP routing is used in the IP network to determine the appropriate point of termination (in this instance a gateway) and to establish a SIP dialog and begin negotiation of a media session between the origination and termination endpoints. The egress gateway then signals ISUP to the PSTN, reusing any encapsulated ISUP present in the SIP request it receives as appropriate. 
A very elementary call-flow for SIP bridging is shown below.

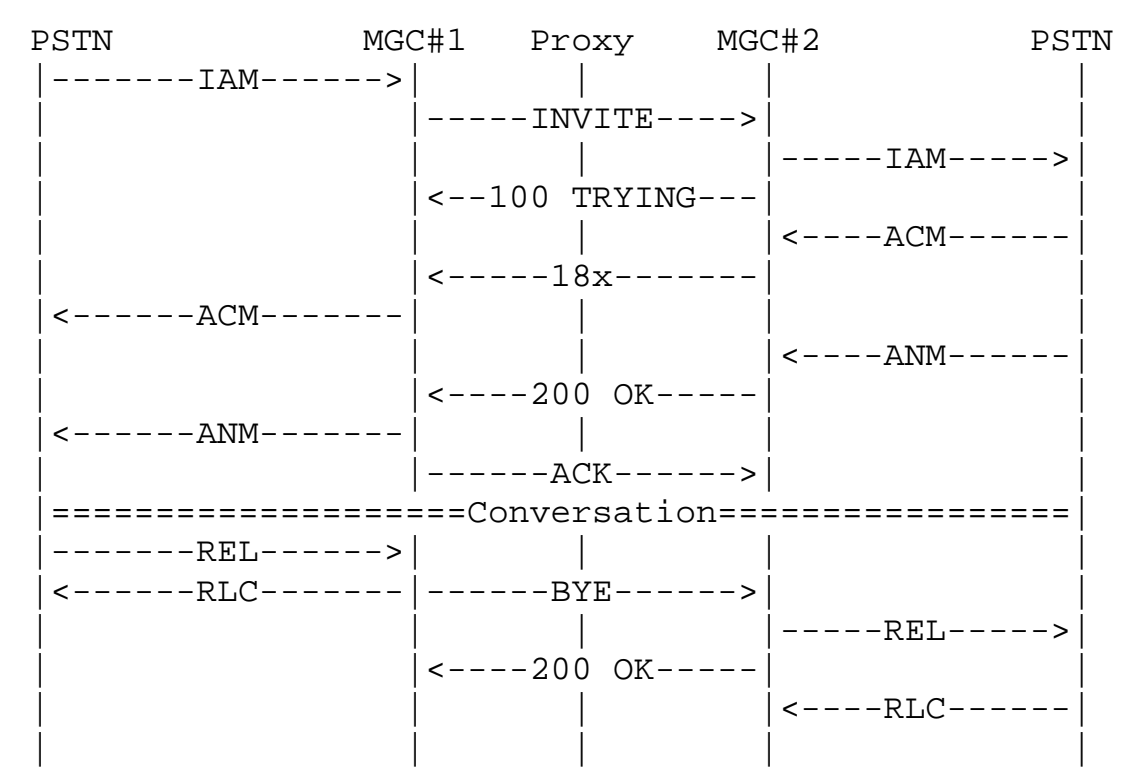

3.2 PSTN origination - IP termination

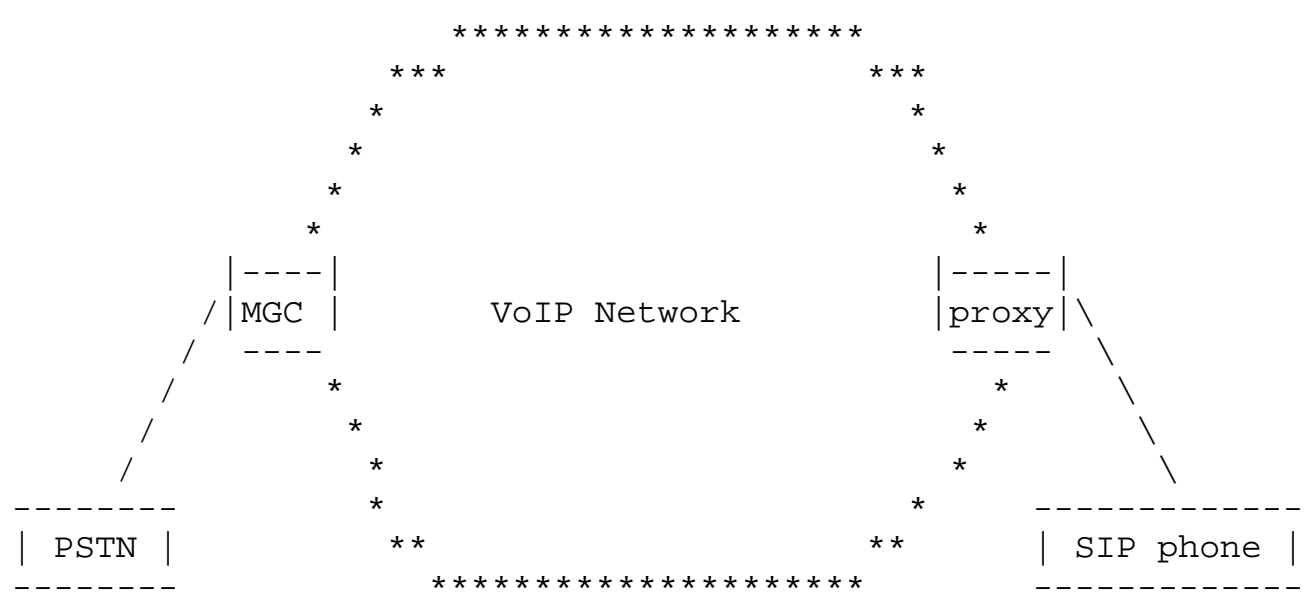

Figure 3: PSTN origination - IP termination 
A call originates from the PSTN and terminates at a SIP phone. Note that in Figure 5, the proxy server acts as the registrar for the SIP phone in question.

A simple call-flow depicting the ISUP and SIP signaling for a PSTNoriginated call terminating at a SIP endpoint follows:

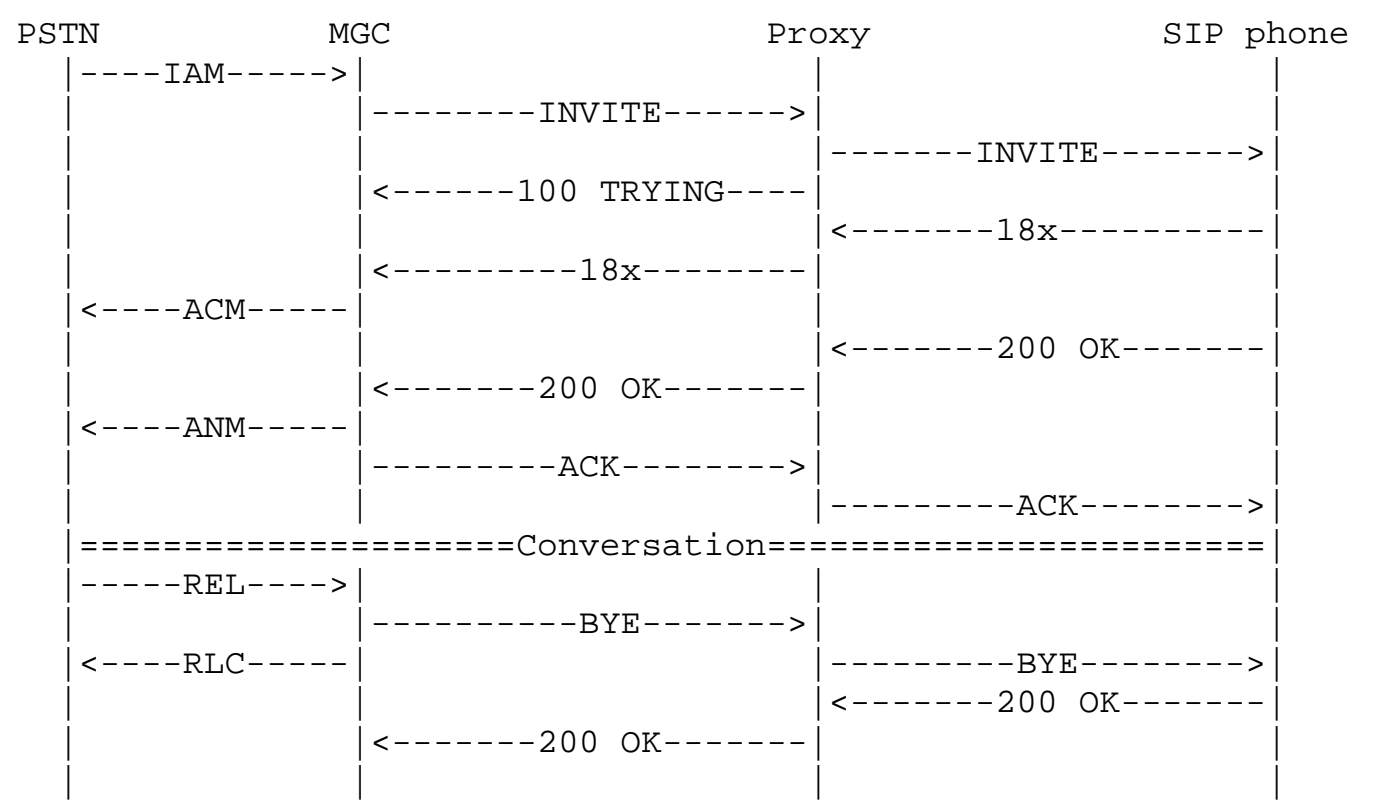


3.3 IP origination - PSTN termination

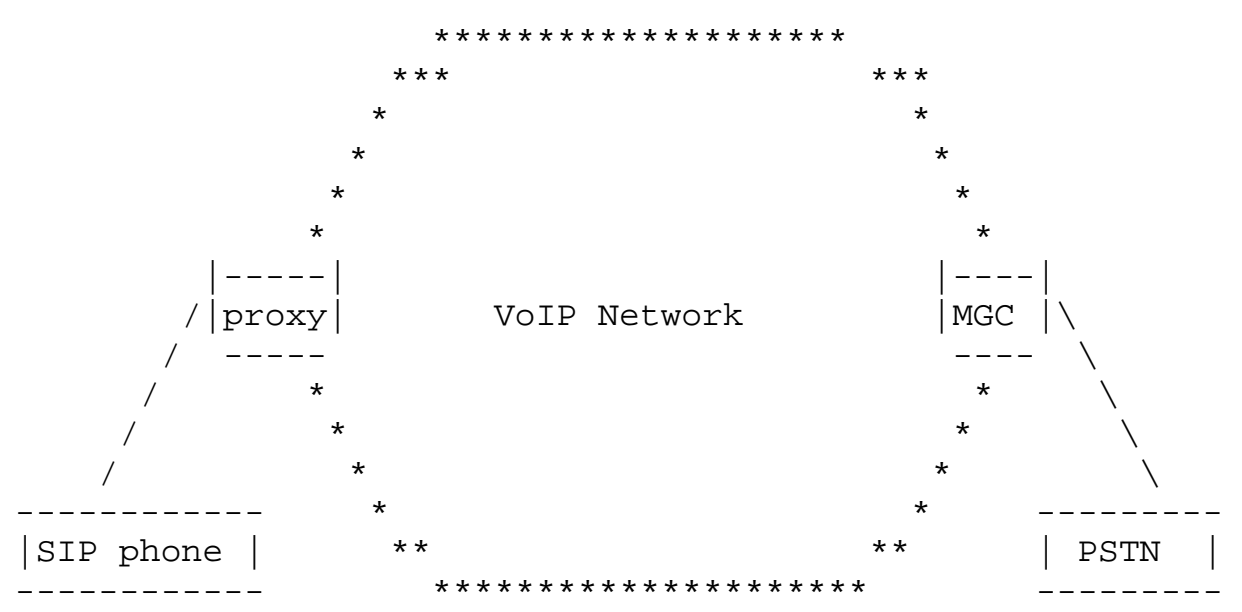

Figure 4: IP origination - PSTN termination

A call originates from a SIP phone and terminates in the PSTN.

Unlike the previous two flows, there is therefore no ISUP

encapsulation in the request - the terminating gateway therefore only performs translation on the SIP headers to derive values for ISUP parameters.

A simple call-flow illustrating the different legs in the call is as shown below. 


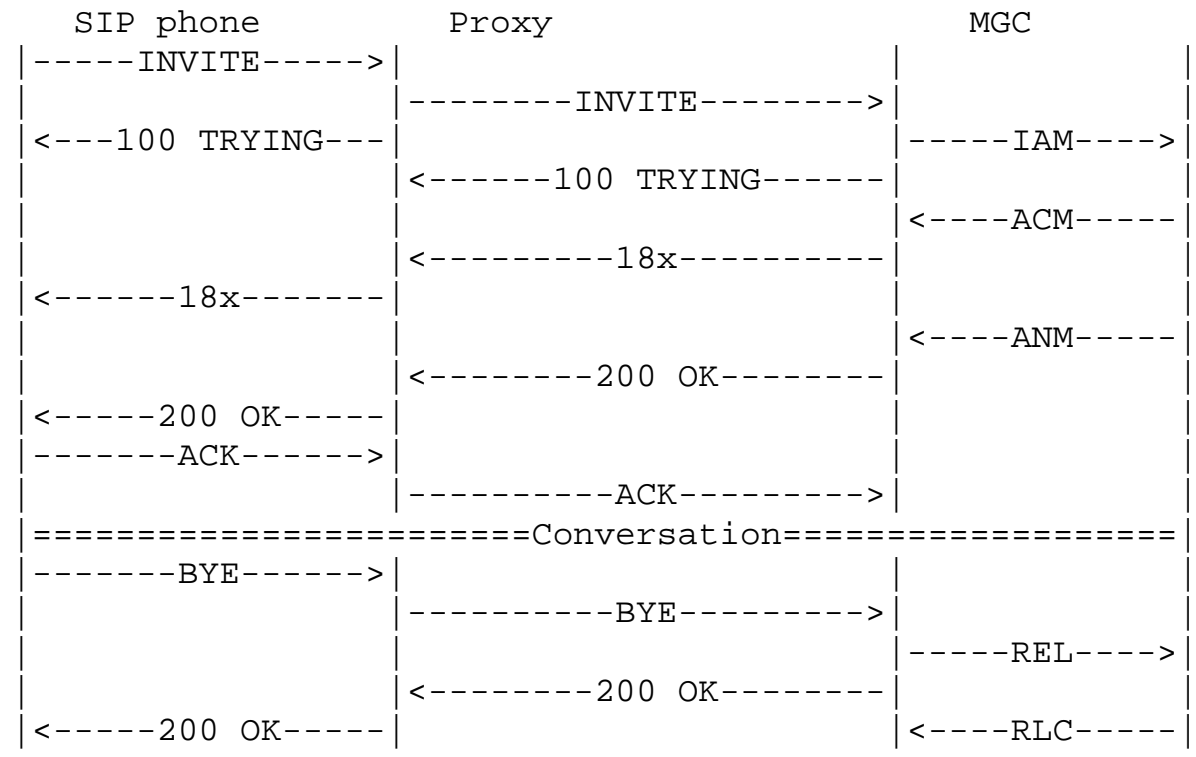

4. SIP-T Roles and Behavior

There are three distinct sorts of elements (from a functional point of view) in a SIP VoIP network that interconnects with the PSTN:

1. The originators of SIP signaling

2. The terminators of SIP signaling

3. The intermediaries that route SIP requests from the originator to the terminator

Behavior for the section 4.1, Section 4.2 and section 4.3 intermediary roles in a SIP-T call are described in the following sections.

\subsection{Originator}

The function of the originating user agent client is to generate the SIP Call setup requests (i.e., INVITEs). When a call originates in the PSTN, a gateway is the UAC; otherwise some native SIP endpoint is the UAC. In either case, note that the originator generally cannot anticipate what sort of entity the terminator will be, i.e., whether final destination of the request is in a SIP network or the PSTN. 
In the case of calls originating in the PSTN (see Figure 3 and Figure 5), the originating gateway takes the necessary steps to preserve the ISUP information by encapsulating it in the SIP request it creates. The originating gateway is entrusted with the responsibility of identifying the version of the ISUP (ETSI, ANSI, etc.) that it has received and providing this information in the encapsulated ISUP (usually by adding a multipart MIME body with appropriate MIME headers). It then formulates the headers of the SIP INVITE request from the parameters of the ISUP that it has received from the PSTN as appropriate (see Section 5). This might, for instance, entail setting the 'To:' header field in the INVITE to the reflect dialed number (Called Party Number) of the received ISUP IAM.

In other cases (like Figure 7), a SIP phone is the originator of a VoIP call. Usually, the SIP phone sends requests to a SIP proxy that is responsible for routing the request to an appropriate destination. There is no ISUP to encapsulate at the user agent client, as there is no PSTN interface. Although the call may terminate in the telephone network and need to signal ISUP in order for that to take place, the originator has no way to anticipate this and it would be foolhardy to require that all SIP VoIP user agents have the capability to generate ISUP. It is therefore not the responsibility of an IP endpoints like a SIP phone to generate encapsulated ISUP. Thus, an originator must generate the SIP signaling while performing ISUP encapsulation and translation when possible (meaning when the call has originated in the PSTN).

Originator requirements: encapsulate ISUP, translate information from ISUP to SIP, multipart MIME support (for gateways only)

\subsection{Terminator}

The SIP-T terminator is a consumer of the SIP calls. The terminator is a standard SIP UA that can be either a gateway that interworks with the PSTN or a SIP phone. 
In case of PSTN terminations (see Figure 3 and Figure 7) the egress gateway terminates the call to its PSTN interface. The terminator generates the ISUP appropriate for signaling to the PSTN from the incoming SIP message. Values for certain ISUP parameters may be gleaned from the SIP headers or extracted directly from an encapsulated ISUP body. Generally speaking, a gateway uses any encapsulated ISUP as a template for the message it will send, but it overwrites parameter values in the template as it translates SIP headers or adds any parameter values that reflect its local policies (see Appendix A item 1).

In case of an IP termination (Figure 5), the SIP UAS that receives SIP messages with encapsulated ISUP typically disregards the ISUP message. This does introduce a general requirement, however, that devices like SIP phones handle multipart MIME messages and unknown MIME types gracefully (this is a baseline SIP requirement, but also a place where vendors have been known to make shortcuts).

Terminator requirements: standard SIP processing, interpretation of encapsulated ISUP (for gateways only), support for multipart MIME, graceful handling of unknown MIME content (for non-gateways only)

\subsection{Intermediary}

Intermediaries like proxy servers are entrusted with the task of routing messages to one another, as well as gateways and SIP phones. Each proxy server makes a forwarding decision for a SIP request based on values of various headers, or 'routable elements' (including the Request-URI, route headers, and potentially many other elements of a SIP request).

SIP-T does introduce some additional considerations for forwarding a request that could lead to new features and requirements for intermediaries. Feature transparency of ISUP is central to the notion of SIP-T. Compatibility between the ISUP variants of the originating and terminating PSTN interfaces automatically leads to feature transparency. Thus, proxy servers might take an interest in the variants of ISUP that are encapsulated with requests - the variant itself could become a routable element. The termination of a call at a point that results in greater proximity to the final destination (rate considerations) is also an important consideration. The preference of one over the other results in a trade-off between simplicity of operation and cost. The requirement of procuring a reasonable rate may dictate that a SIP-T call spans dissimilar PSTN interfaces (SIP bridging across different gateways that don't support any ISUP variants in common). In order to optimize for maximum feature transparency and rate, some operators of intermediaries might want to consider practices along the following lines: 
a) The need for ISUP feature transparency may necessitate ISUP variant translation (conversion), i.e., conversion from one variant of ISUP to another in order to facilitate the termination of that call over a gateway interface that does not support the ISUP variant of the originating PSTN interface. (See Appendix A item 2.) Although in theory conversion may be performed at any point in the path of the request, it is optimal to perform it at a point that is at the greatest proximity to the terminating gateway. This could be accomplished by delivering the call to an application that might perform the conversion between variants. Feature transparency in this case is contingent on the availability of resources to perform ISUP conversion, and it incurs an increase in the call-set up time.

b) An alternative would be to sacrifice ISUP transparency by handing the call off to a gateway that does not support the version of the originating ISUP. The terminating MGC would then just ignore the encapsulated ISUP and use the information in the SIP header to terminate the call.

So, it may be desirable for proxy servers to have the intelligence to make a judicious choice given the options available to it.

Proxy requirements: ability to route based on choice of routable elements

\subsection{Behavioral Requirements Summary}

If the SIP-T originator is a gateway that received an ISUP request, it must always perform both encapsulation and translation ISUP, regardless of where the originator might guess that the request will terminate.

If the terminator does not understand ISUP, it ignores it while performing standard SIP processing. If the terminator does understand ISUP, and needs to signal to the PSTN, it should reuse the encapsulated ISUP if it understands the variant. The terminator should perform the following steps:

- Extract the ISUP from the message body, and use this ISUP as a message template. Note that if there is no encapsulated ISUP in the message, the gateway should use a canonical template for the message type in question (a pre-populated ISUP message configured in the gateway) instead. 
- Translate the headers of the SIP request into ISUP parameters, overwriting any values in the message template.

- Apply any local policies in populating parameters.

An intermediary must be able to route a call based on the choice of routable elements in the SIP headers.

5. Components of the SIP-T Protocol

The mechanisms described in the following sections are the components of SIP-T that provide the protocol functions entailed by the requirements.

\subsection{Core SIP}

SIP-T uses the methods and procedures of SIP as defined by RFC 3261.

\subsection{Encapsulation}

Encapsulation of the PSTN signaling is one of the major requirements of SIP-T. SIP-T uses multipart MIME bodies to enable SIP messages to contain multiple payloads (Session Description Protocol or SDP [5], ISUP, etc.). Numerous ISUP variants are in existence today; the ISUP MIME type enable recipients too recognize the ISUP type (and thus determine whether or not they support the variant) in the most expeditious possible manner. One scheme for performing ISUP encapsulation using multi-part MIME has been described in [2] .

\subsection{Translation}

Translation encompasses all aspects of signaling protocol conversion between SIP and ISUP. There are essentially two components to the problem of translation:

1. ISUP SIP message mapping: This describes a mapping between ISUP and SIP at the message level. In SIP-T deployments gateways are entrusted with the task of generating a specific ISUP message for each SIP message received and vice versa. It is necessary to specify the rules that govern the mapping between ISUP and SIP messages (i.e., what ISUP messages is sent when a particular SIP message is received: an IAM must be sent on receipt of an INVITE, a REL for BYE, and so on). A potential mapping between ISUP and SIP messages has been described in [10]. 
2. ISUP parameter-SIP header mapping: A SIP request that is used to set up a telephone call should contain information that enables it to be appropriately routed to its destination by proxy servers in the SIP network - for example, the telephone number dialed by the originating user. It is important to standardize a set of practices that defines the procedure for translation of information from ISUP to SIP (for example, the Called Party Number in an ISUP IAM must be mapped onto the SIP 'TO' header field and Request-URI, etc.). This issue becomes inherently more complicated by virtue of the fact that the headers of a SIP request (especially an INVITE) may be transformed by intermediaries, and that consequently, the SIP headers and encapsulated ISUP bodies come to express conflicting values effectively, a part of the encapsulated ISUP may be rendered irrelevant and obsolete.

\subsection{Support for mid-call signaling}

Pure SIP does not have any provision for carrying any mid-call control information that is generated during a session. The INFO [3] method should be used for this purpose. Note however that INFO is not suitable for managing overlap dialing (for one way of implementing overlap dialing see [11]). Also note that the use of INFO for signaling mid-call DTMF signals is not recommended (see RFC2833 [9] for a recommended mechanism).

6. SIP Content Negotiation

The originator of a SIP-T request might package both SDP and ISUP elements into the same SIP message by using the MIME multipart format. Traditionally in SIP, if the terminating device does not support a multipart payload (multipart/mixed) and/or the ISUP MIME type, it would then reject the SIP request with a 415 unsupported Media Type specifying the media types it supports (by default, 'application/SDP'). The originator would subsequently have to resend the SIP request after stripping out the ISUP payload (i.e. with only the SDP payload) and this would then be accepted.

This is a rather cumbersome flow, and it is thus highly desirable to have a mechanism by which the originator could signify which bodies are required and which are optional so that the terminator can silently discard optional bodies that it does not understand (allowing a SIP phone to ignore an ISUP payload when processing ISUP is not critical). This is contingent upon the terminator having support for a Content-type of multipart/mixed and access to the Content-Disposition header to express criticality. 
1. Support for ISUP is optional. Therefore, UA2 accepts the INVITE irrespective of whether it can process the ISUP.

UA1

UA2

INVITE-->

(Content-type:multipart/mixed;

Content-type: application/sdp;

Content-disposition: session; handling=required;

Content-type: application/isup;

Content-disposition: signal; handling=optional;)

$$
<--18 x
$$

2. Support for ISUP is preferred. UA2 does not support the ISUP and rejects the INVITE with a 415 Unsupported Media Type. UA1 strips off the ISUP and re-sends the INVITE with SDP only and this is the accepted.

UA1 UA2

INVITE--> (Content-type:multipart/mixed;

Content-type: application/sdp;

Content-disposition: session; handling=required;

Content-type: application/isup;

Content-disposition: signal; handling=required; )

$$
<--415
$$

(Accept: application/sdp)

ACK $-->$

INVITE-->

(Content-type: application/sdp)

$$
<--18 x
$$

3. Support for ISUP is mandatory for call establishment. UA2 does not support the ISUP and rejects the INVITE with a 415 Unsupported Media type. UA1 then directs its request to UA3. 


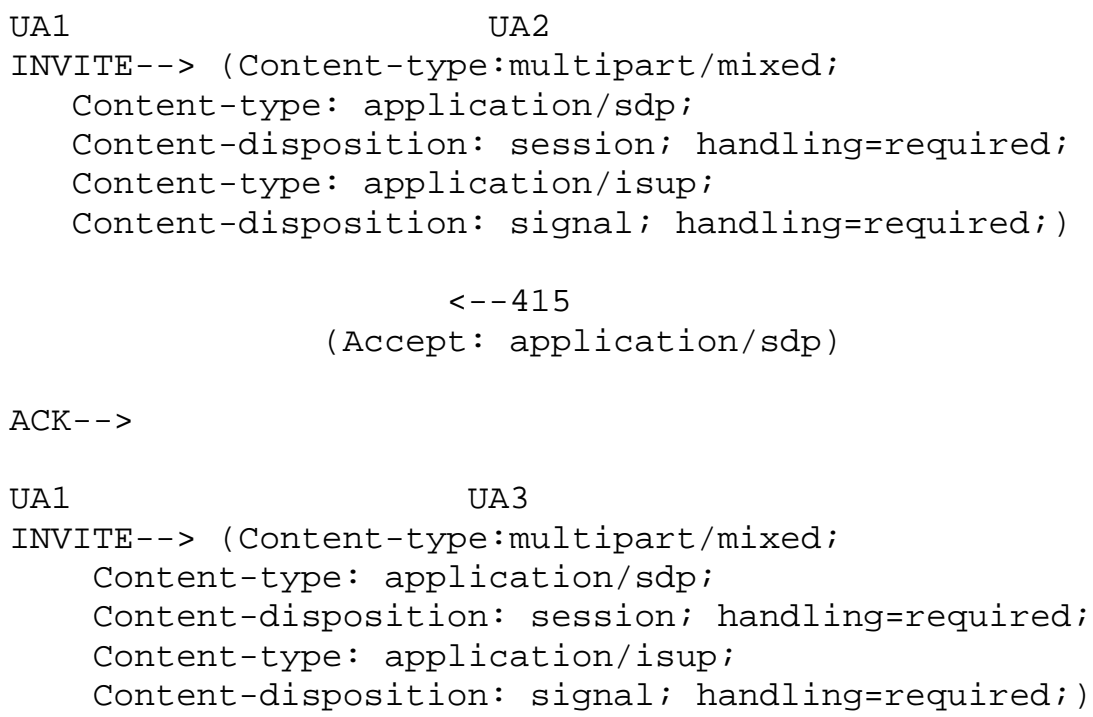

Note that the exchanges of messages above are not complete; only the messages relevant to this discussion are shown. Specifics of the ISUP MIME type can be obtained from [2]. The 'version' and 'base' parameters are not shown here, but must be used in accordance with the rules of [2].

7. Security Considerations

SIP-T can be employed as an interdomain signaling mechanism that may be subject to pre-existing trust relationships between administrative domains. In many legal environments, distribution of ISUP is restricted to licensed carriers; SIP-T introduces some challenges in so far as it bridges carrier signaling with end-user signaling. Any administrative domain implementing SIP-T should have an adequate security apparatus (including elements that manage any appropriate policies to manage fraud and billing in an interdomain environment) in place to ensure that the transmission of ISUP information does not result in any security violations.

Transporting ISUP in SIP bodies may provide opportunities for abuse, fraud, and privacy concerns, especially when SIP-T requests can be generated, inspected or modified by arbitrary SIP endpoints. ISUP MIME bodies should be secured (preferably with S/MIME [4]) to alleviate this concern, as is described in the security Considerations of the core SIP specification [1]. Authentication properties provided by S/MIME would allow the recipient of a SIP-T message to ensure that the ISUP MIME body was generated by an 
authorized entity. Encryption would ensure that only carriers possessing a particular decryption key are capable of inspecting encapsulated ISUP MIME bodies in a SIP request.

SIP-T endpoints MUST support S/MIME signatures (CMS SignedData), and SHOULD support encryption (CMS EnvelopedData).

8. IANA Considerations

This document introduces no new considerations for IANA.

\section{Normative References}

[1] Rosenberg, J., Schulzrinne, H., Camarillo, G., Johnston, A., Peterson, J., Sparks, R., Handley, M. and E. Schooler, "SIP: Session Initiation Protocol", RFC 3261, May 2002.

[2] Zimmerer, E., Peterson, J., Vemuri, A., Ong, L., Audet, F., Watson, M. and M. Zonoun, "MIME media types for ISUP and QSIG objects", RFC 3204, December 2001.

[3] Donovan, S., "The SIP INFO Method", RFC 2976, October 2000.

[4] Ramsdell, B., "S/MIME Version 3 Message Specification", RFC 2633, June 1999.

[5] Handley, M. and V. Jacobson, "SDP: Session Description Protocol", RFC 2327, April 1998.

Non-Normative References

[6] International Telecommunications Union, "Signaling System No. 7; ISDN User Part Signaling procedures", ITU-T Q.764, September 1997, <http://www.itu.int>.

[7] Faltstrom, P., "E.164 number and DNS", RFC 2916, September 2000 .

[8] Rosenberg, J., Salama, H. and M. Squire, "Telephony Routing over IP (TRIP)", RFC 3219, January 2002.

[9] Schulzrinne, H. and S. Petrack, "RTP Payload for DTMF Digits, Telephony Tones and Telephony Signals", RFC 2833, May 2000.

[10] Camarillo, G., Roach, A., Peterson, J. and L. Ong, "ISUP to SIP Mapping", Work in Progress. 
[11] Camarillo, G., Roach, A., Peterson, J. and L. Ong, "Mapping of ISUP Overlap Signaling to SIP", Work in Progress. 
Appendix A. Notes

1. Some terminating MGCs may alter the encapsulated ISUP in order to remove any conditions specific to the originating circuit; for example, continuity test flags in the Nature of Connection Indicators, etc.

2. Even so, the relevance of ANSI-specific information in an ETSI network (or vice versa) is questionable. Clearly, the strength of SIP-T is realized when the encapsulated ISUP involves the usage of proprietary parameters.

Appendix B. Acknowledgments

We thank Andrew Dugan, Rob Maidhof, Dave Martin, Adam Roach, Jonathan Rosenberg, Dean Willis, Robert F. Penfield, Steve Donovan, Allison Mankin, Scott Bradner and Steve Bellovin for their valuable comments.

The original 'SIP+' proposal for interconnecting portions of the PSTN with SIP bridging was developed by Eric Zimmerer.

Authors' Addresses

Aparna Vemuri-Pattisam

Qwest Communications

6000 Parkwood Pl

Dublin, OH 43016 US

EMail: Aparna.Vemuri@Qwest.com vaparna10@yahoo.com

Jon Peterson

NeuStar, Inc.

1800 sutter st

Suite 570

Concord, CA 94520 US

Phone: +1 925/363-8720

EMail: jon.peterson@neustar.biz

URI: http://www.neustar.biz/ 
Full Copyright statement

Copyright (C) The Internet Society (2002). All Rights Reserved.

This document and translations of it may be copied and furnished to others, and derivative works that comment on or otherwise explain it or assist in its implementation may be prepared, copied, published and distributed, in whole or in part, without restriction of any kind, provided that the above copyright notice and this paragraph are included on all such copies and derivative works. However, this document itself may not be modified in any way, such as by removing the copyright notice or references to the Internet Society or other Internet organizations, except as needed for the purpose of developing Internet standards in which case the procedures for copyrights defined in the Internet standards process must be followed, or as required to translate it into languages other than English.

The limited permissions granted above are perpetual and will not be revoked by the Internet Society or its successors or assigns.

This document and the information contained herein is provided on an "AS IS" basis and THE INTERNET SOCIETY AND THE INTERNET ENGINEERING TASK FORCE DISCLAIMS ALL WARRANTIES, EXPRESS OR IMPLIED, INCLUDING BUT NOT LIMITED TO ANY WARRANTY THAT THE USE OF THE INFORMATION HEREIN WILL NOT INERINGE ANY RIGHTS OR ANY IMPLIED WARRANTIES OF MERCHANTABILITY OR FITNESS FOR A PARTICULAR PURPOSE.

Acknowledgement

Funding for the RFC Editor function is currently provided by the Internet Society. 
